\title{
Assessment of 4D MR Angiography at 3T Compared with DSA for the Follow-up of Embolized Brain Dural Arteriovenous Fistula: A Dual-Center Study
}

\author{
(D)B. Dissaux, (D)F. Eugène, (D). Ognard, (D).-Y. Gauvrit, (D).-C. Gentric, and (D).-C. Ferré
}

\begin{abstract}
BACKGROUND AND PURPOSE: 4D contrast-enhanced MRA in the follow-up of treated dural arteriovenous fistulas has rarely been evaluated. Our aim was to evaluate its diagnostic performance at $3 \mathrm{~T}$ in the follow-up of embolized dural arteriovenous fistulas using DSA as the standard of reference.
\end{abstract}

\begin{abstract}
MATERIALS AND METHODS: Patients treated for dural arteriovenous fistulas in 2 centers between 2008 and 2019 were included if they met the following criteria: 1) dural arteriovenous fistula embolization, and 2) follow-up imaging with $<6$ months between DSA and 4D contrast-enhanced MRA. Two readers reviewed the 4D contrast-enhanced MRA images, first independently, then in consensus to detect any residual/recurrent dural arteriovenous fistula and to grade cases according to the Cognard classification system. Interobserver and intermodality agreement for the detection of a residual dural arteriovenous fistula and stratification of bleeding risk (0-I-IIa; IIb-IIa+b-III-IV-V) was calculated using $\kappa$ coefficients.
\end{abstract}

RESULTS: A total of 51 pairs of examinations for 44 patients (median age, 65 years; range, 25-81 years) were analyzed. Interobserver agreement for the detection and stratification of bleeding risk was, respectively, $\kappa=0.8(95 \% \mathrm{Cl}, 0.6-1)$ and $\kappa=0.8(95 \% \mathrm{Cl}, 0.5-$ 1). After consensus review, the sensitivity and specificity of $4 \mathrm{D}$ contrast-enhanced MRA for the detection of residual/recurrent dural arteriovenous fistula was $63.6 \%(95 \% \mathrm{Cl}, 40.7 \%-82.8 \%)$ and $96.6 \%(95 \% \mathrm{Cl}, 82.2 \%-99.9 \%)$, respectively. The positive and negative predictive values of 4D contrast-enhanced MRA were $93.3 \%(95 \% \mathrm{Cl}, 68.1 \%-99.8 \%)$ and $77.8 \%(95 \% \mathrm{Cl}, 60.8 \%-89.9 \%)$. Intermodality agreement for the detection and stratification of bleeding risk was good, with $\kappa=0.60(95 \% \mathrm{Cl}, 0.3-0.8)$.

CONCLUSIONS: 4D contrast-enhanced MRA at 3T is of interest in the follow-up of treated dural arteriovenous fistulas but lacks the sensitivity to replace arteriography.

ABBREVIATIONS: DAVF = dural arteriovenous fistula; 4D-MRA $=4 \mathrm{D}$ contrast-enhanced magnetic resonance angiography

D ural arteriovenous fistulas (DAVFs) are abnormal arteriovenous connections between dural vessels. The risk of intracranial hemorrhage is variable according to the venous drainage patterns. ${ }^{1-5}$ There are several treatment options, including surgical resection and endovascular embolization, which can be attempted to achieve a cure. The risk of bleeding persists as long as an anatomic cure is not completely achieved, with risk depending on the residual venous drainage pattern. Therefore, it is

Received March 19, 2020; accepted after revision September 27.

From the Department of Neuroradiology (B.D., F.E., J.-Y.G., J.-C.F.), Centre Hospitalier Universitaire Rennes, Rennes, France; Department of Medical Imaging (B.D., J.O., J.-C.G.), Centre Hospitalier Universitaire La Cavale Blanche, Brest, France; GETBO group EA3878 (B.D., J.-C.G.), Université de Bretagne Occidentale, Brest, France; LATIM U1101 (J.O.), INSERM, Université de Bretagne Occidentale, Brest, France; Empenn Unit U1228 (J.-Y.G., J.-C.F.), INSERM, INRIA, Université Rennes 1 Rennes, France.

Please address correspondence to Brieg Dissaux, MD, Service d'Imagerie Médicale CHU de la Cavale Blanche, Boulevard Tanguy-Prigent, 29609 Brest cedex, France; e-mail: brieg.dissaux@chu-brest.fr

http://dx.doi.org/10.3174/ajnr.A6903 necessary to confirm that the DAVF has been effectively cured after treatment.

Due to its high sensitivity and specificity, DSA is the current method of choice in the diagnosis and follow-up of DAVFs despite several disadvantages, such as radiation exposure for patients and medical staff, injection risk of iodinated contrast agent (including allergy and nephrotoxicity), and neurologic procedural risks $(0.30 \%-2.63) .{ }^{1,6}$ The technique has very good spatial and, especially, temporal resolution, allowing precise evaluation of a potential residual shunt.

Several noninvasive cross-sectional imaging techniques such as $3 \mathrm{D}-$ TOF-MRA and $3 \mathrm{D}$ contrast-enhanced MRA have been used to reduce the risk of invasive procedures for patients who otherwise would undergo repeat angiography during treatment planning or follow-up. The diagnostic accuracy of these techniques has proved to be relatively good, but not sufficient to replace DSA due to limited spatial resolution and a static temporal view without temporal hemodynamic information, such as arterial 
phase venous filling. ${ }^{3}$ 4D contrast-enhanced MRA (4D-MRA) was conceived to solve this problem and provide better temporal resolution while also preserving spatial resolution. With improving technology, it became a widely used technique with the advantage of a dynamic DSA-like evaluation of DAVFs. Previous studies report time-resolved 3T MR angiography as an appropriate tool for DAVF diagnosis and monitoring. However, the value of 4D-MRA for the follow-up of patients with treated DAVFs has rarely been evaluated.

We hypothesized that this technique could be valuable for the follow-up and posttherapeutic assessment of DAVFs. With DSA images as the standard of reference, the purpose of this study was to evaluate the performance of $4 \mathrm{D}-\mathrm{MRA}$ at $3 \mathrm{~T}$ in the follow-up of patients with treated DAVFs.

\section{MATERIALS AND METHODS \\ Study Design}

Institutional review board approval was granted (No. 19.87), and informed consent was waived due to the study design. All patients treated for DAVFs in 2 university hospital departments (Rennes and Brest, France) were included in a data base. For this study, patients imaged between August 2008 and May 2019 were included if they met the following criteria:

1. They had a DAVF treated with embolization

2. They underwent both $4 \mathrm{D}-\mathrm{MRA}$ at $3 \mathrm{~T}$ and DSA during follow-up

3. Both examinations were performed within a 6-month interval without treatment between them.

\section{Treatment Strategy}

For all patients, the indication and strategy of treatment were based on multidisciplinary decisions involving neurologists, neurosurgeons, and neuroradiologists.

\section{MR Imaging}

All MRA examinations were performed on a 3T MR imaging system (Achieva and Ingenia, Philips Healthcare, Best, Netherlands). All contrast-enhanced 4D-MRA examinations consisted of coronal, sagittal, and axial MIP subtraction images derived from a sagittal time-resolved 3D T1-weighted fast gradient-echo sequence. Several acquisition schemes were used according to the brain coverage (full or two-thirds). At least 20 dynamic acquisitions were performed with a temporal resolution of $0.9-$ 1.7 seconds per volume and a native spatial resolution from $0.8 \times$ $0.8 \times 1.6 \mathrm{~mm}^{3}$ to $1.1 \times 1.1 \times 2.8 \mathrm{~mm}^{3}$ and, after interpolation, ranging from $0.5 \times 0.5 \times 0.9 \mathrm{~mm}^{3}$ to $0.94 \times 0.94 \times 1.4 \mathrm{~mm}^{3}$. The 15- mL macrocyclic gadolinium bolus was administered intravenously at a minimum rate of $3 \mathrm{~mL} / \mathrm{s}^{7}$

\section{DSA Technique}

DSA was performed on a biplane angiography system (Allura, Philips Healthcare, Best, Netherlands and Artis, Siemens Healthineers, Erlangen, Germany). DSA images involved selective injection of internal and external carotid and vertebral arteries with anterior-posterior and lateral projections, supplemented by additional views when necessary. Each projection was acquired with a frequency of 2-3 images per second. For each projection, a 6- to $10-\mathrm{mL}$ bolus of nonionic iodinated contrast material was injected with a power injector.

\section{End Points}

The primary end point was to evaluate the diagnostic reproducibility and performance of 4D-MRA for detecting any residual/ recurrent shunts in patients with treated DAVFs using DSA as the standard of reference. The secondary end point was to evaluate the diagnostic reproducibility and performance of $4 \mathrm{D}-\mathrm{MRA}$ for detecting any high-bleeding-risk residual/recurrent shunts in patients with treated DAVFs using DSA as the standard of reference.

\section{Interpretation}

Readers were blinded to all clinical data except the original location of the treated DAVF. Only the 3 MIPs were used to read the 4D-MRA. One reader (J.-C.F., reader 3) with 17 years' experience in neuroradiology reviewed the DSA images. Two readers (F.E., reader 1 , and B.D., reader 2) with 9 and 5 years' experience in neuroradiology, respectively, reviewed the 4D-MRA images. First, readers 1 and 2 assessed the quality of the 4D-MRA images. 4D MRA image-quality scores ranged among 0 (no vascular study possible), 1 (vascular study possible with low diagnostic confidence), 2 (vascular study possible with adequate diagnostic confidence), and 3 (vascular study possible with high diagnostic confidence). The readers independently assessed the presence of residual/recurrent DAVFs on the $4 \mathrm{D}-\mathrm{MRA}$ and DSA images. Then, when present, each DAVF was graded according to the Cognard classification scheme and divided into 2 groups based on bleeding risk: low-bleeding-risk DAVFs (types I and IIa) and high-bleeding-risk DAVFs (types IIb, IIa + b, III, IV, and V). ${ }^{5}$ Second, a consensus reading (readers 1 and 2) of the 4D-MRA images was conducted to solve any discrepancies. Third, a retrospective explanatory analysis was performed in consensus by the 3 readers to explain intermodality (4D-MRA and DSA) differences.

\section{Data Analysis and Sample Size}

Baseline characteristics, including age and fistula type, were summarized using descriptive statistics. The intervals among DSA and 4D-MRA, treatment, and the first imaging technique (DSA or 4D-MRA) were recorded. 4D-MRA interobserver and intermodality agreement was assessed using $\kappa$ for the following: 1) residual/recurrent DAVF detection, and 2) bleeding-risk grading with a $2 \times 2$ contingency table (low-risk: absence of a shunt and Cognard types I-IIa; high-risk: Cognard types IIb, III, IV, and V) and on a 3-tier modified scale (comprising absence of a shunt; Cognard types I-IIa; and Cognard types IIb, III, IV, and V). The Cohen $\kappa$ coefficient was calculated using quadratic weighting (for bleeding-risk grading with the 3-tier modified scale). The $95 \%$ confidence intervals for $\kappa$ were estimated with the bootstrap method. $\kappa$ statistics were interpreted as suggested by Landis and Koch $(\kappa<0$, poor agreement; $0.01-0.20$, slight agreement; $0.21-$ 0.40 , fair agreement; $0.41-0.60$, moderate agreement; $0.61-0.80$, substantial agreement; and $0.81-1$, almost perfect agreement).

The binary decision regarding the presence of a residual/ recurrent DAVF and a residual high-bleeding-risk DAVF (low 
risk: absence of a shunt and Cognard types I-IIa; high risk: Cognard types IIb, III, IV, and V) was used to determine the diagnostic performance of 4D-MRA (sensitivity, specificity, positive predictive value, negative predictive value, the area under the ROC curve), and 95\% confidence intervals were estimated with generalized estimating equations.

All statistical analyses were performed using STATA software (STATA/MP 16.0; StataCorp).

\section{RESULTS}

We included 51 examination pairs (4D-MRA and DSA within 6 months) for 44 patients with a median age of 65 years (range, 25-81 years). No examination (neither 4D-MRA nor DSA) was included $>1$ time. Nineteen (43\%) of the 44 patients were women. Pretreatment DAVF grading was as follows-type I: $n=1(2.3 \%)$, type IIa: $n=2(4.6 \%)$, type IIb: $n=13(29.5 \%)$, type IIa + b: $n=0(0 \%)$, type III: $n=13$ (29.5\%), type IV: $n=12(27.3 \%)$, and type V: $n=3(6.8 \%)$. Thirty-nine $(88.6 \%)$ patients underwent 1 embolization session, and 5 patients (11.4\%) underwent 2 sessions. The corresponding flowchart is shown in Fig 1. The median interval between $4 \mathrm{D}$-MRA and DSA was 49 days (range, 0-155 days). 4D-MRA was performed before DSA in 31 (70.4\%) examination pairs. The median interval between DAVF treatment and the first examination was 124 days (range, 2-730 days). The DSA images yielded the following results-no residual/

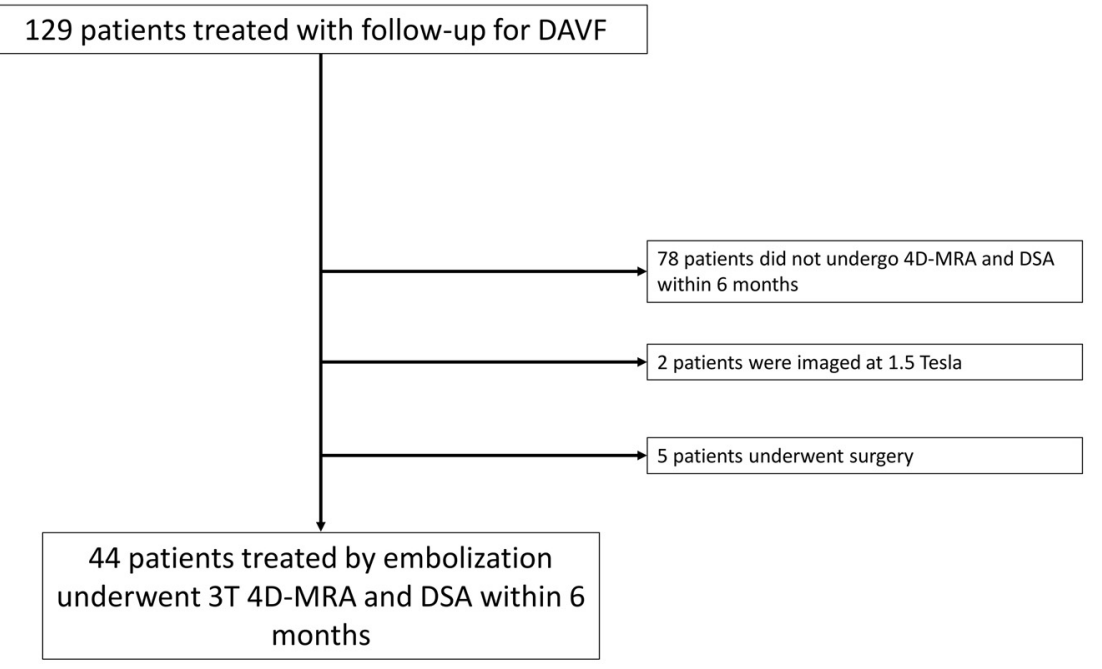

FIG 1. Flow chart. recurrent DAVF: $29 / 51$ (56.9\%), type I: $n=4$ (7.9\%), type IIa: $n=4(7.9 \%)$, type IIb: $n=1(1.9 \%)$, type IIa + b: $n=0$ (0\%), type III: $n=11(21.6 \%)$, type IV: $n=1$ (1.9\%), and type $\mathrm{V}$ residual/recurrent DAVF: $n=1$ (1.9\%). Twelve examination pairs were obtained from 5 patients: After the first follow-up $(n=5), 4$ examination pairs were obtained after a new treatment and 3 later in the follow-up of the first treatment.

\section{D-MRA Image Quality}

After reaching a consensus, all images were given a minimum score of 1 . The median quality score was 3 . One (1.9\%) 4D-MRA examination scored 1, seven (13.7\%) scored 2 , and $43(84.4 \%)$ scored 3 on the 4 -point grading scale, respectively.

\section{D-MRA Interobserver Agreement}

One reader assigned 1 examination a score of zero (no vascular study possible). Fifty examinations were assessed for interobserver agreement. Interobserver agreement was considered substantial for residual/recurrent DAVF detection, with an agreement of $92 \%$ and $\kappa=0.8$ (95\% CI, 0.6-1). Disagreement among readers (readers 1 and 2) is shown in Table 1. Interobserver agreement was considered substantial in terms of the ability to detect a fistula at risk of hemorrhage, with an agreement of $92 \%$ and $\kappa=0.8$ (95\% CI, $0.5-1)$ in a 2 $\times 2$ contingency table (low-risk: absence of a shunt and types I-IIa; high-risk: types IIb, III, IV, and $\mathrm{V}$ ) and an agreement of $92 \%$ and $\kappa=0.8$ (95\% CI, 0.8-0.9) on a 3-tier modified scale (comprising absence of a shunt; types I-IIa; and IIb, III, IV, and V).

\section{Intermodality Agreement}

After reaching a 4D-MRA reading consensus, 51 comparisons were available. Table 2 provides the results for the 2 modalities. Disagreement between readers 1 and 2 is shown in Table 3.

Intermodality agreement was considered substantial for residual/ recurrent DAVF detection, with an agreement of $82.4 \%$ and $\kappa=0.6(95 \%$ CI, 0.4-0.8). Intermodality agreement

Table 1: Interreader disagreement in 4D-MRA residual/recurrent DAVF classification

\begin{tabular}{|c|c|c|c|c|c|}
\hline $\begin{array}{l}\text { Dural Fistula } \\
\text { Location }\end{array}$ & $\begin{array}{l}\text { 4D-MRA } \\
\text { Classification } \\
\text { Reader } 1\end{array}$ & $\begin{array}{c}\text { 4D-MRA } \\
\text { Classification } \\
\text { Reader } 2\end{array}$ & $\begin{array}{c}\text { 4D-MRA } \\
\text { Classification } \\
\text { Consensus }\end{array}$ & $\begin{array}{c}\text { DSA } \\
\text { Classification }\end{array}$ & $\begin{array}{c}\text { 4D-MRA } \\
\text { Consensus } \\
\text { Quality Score }\end{array}$ \\
\hline Hemispheric right & 0 & III & III & III & 3 \\
\hline Parasagittal right & 0 & III & 0 & 0 & 3 \\
\hline SSS & 0 & Ila & 0 & 0 & 1 \\
\hline Epiphyseal & 0 & III & III & III & 3 \\
\hline Sinus lateralis left & I & 0 & I & I & 3 \\
\hline Medulla & III & 0 & 0 & V & 3 \\
\hline
\end{tabular}

Note:-SSS indicates superior sagittal sinus. 
Table 2: Contingency table of residual/recurrent DAVF grading according to technique (4D-MRA and DSA) ${ }^{a}$

\begin{tabular}{|c|c|c|c|c|c|c|c|c|}
\hline \multirow[b]{2}{*}{ DSA } & \multicolumn{8}{|c|}{ 4D-MRA } \\
\hline & No Shunt & Type $\mathrm{I}^{\mathrm{b}}$ & Type Ilab $^{\mathrm{b}}$ & Type $I I b^{c}$ & Type Ila $+b^{c}$ & Type III ${ }^{\mathrm{C}}$ & Type IV ${ }^{c}$ & Type $\mathrm{V}^{\mathrm{c}}$ \\
\hline No shunt & 28 & 0 & 0 & 0 & 1 & 0 & 0 & 0 \\
\hline Type $1^{b}$ & 1 & 3 & 0 & 0 & 0 & 0 & 0 & 0 \\
\hline Type Ilab & 1 & 0 & 3 & 0 & 0 & 0 & 0 & 0 \\
\hline Type $I I b^{c}$ & 0 & 0 & 1 & 0 & 0 & 0 & 0 & 0 \\
\hline Type Ila $+b^{c}$ & 0 & 0 & 0 & 0 & 0 & 0 & 0 & 0 \\
\hline Type III & 4 & 0 & 0 & 0 & 0 & 4 & 3 & 0 \\
\hline Type IV ${ }^{c}$ & 1 & 0 & 0 & 0 & 0 & 0 & 0 & 0 \\
\hline Type $V^{c}$ & 1 & 0 & 0 & 0 & 0 & 0 & 0 & 0 \\
\hline
\end{tabular}

${ }^{a}$ Consensus grading according to Cognard et al. ${ }^{5}$

b Low bleeding risk.

${ }^{c}$ High bleeding risk.

Table 3: Intermodality discrepancy in residual/recurrent dural arteriovenous fistula detection and classification ${ }^{\mathrm{a}}$

\begin{tabular}{|c|c|c|c|c|c|c|c|c|}
\hline Location & $\begin{array}{l}\text { Pretreatment } \\
\text { Grading }\end{array}$ & $\begin{array}{l}\text { 4D-MRA/ } \\
\text { DSA } \\
\text { Interval } \\
\text { (Days) }\end{array}$ & $\begin{array}{c}\text { DSA } \\
\text { Grading }\end{array}$ & $\begin{array}{l}\text { 4D-MRA } \\
\text { Consensus } \\
\text { Grading }\end{array}$ & $\begin{array}{c}\text { 4D-MRA Reader } \\
\text { Disagreement } \\
\text { (Yes/No) }\end{array}$ & Year & $\begin{array}{l}\text { 4D-MRA } \\
\text { Consensus } \\
\text { Quality } \\
\text { Score }\end{array}$ & Possible Explanation \\
\hline Parasagittal right & III & 65 & IV & 0 & No & 2010 & 3 & $\begin{array}{l}\text { Confusion between } \\
\text { occipital artery and a } \\
\text { cortical vein }\end{array}$ \\
\hline $\begin{array}{l}\text { Cavernous sinus } \\
\text { right }\end{array}$ & III & 59 & III & 0 & No & 2015 & 2 & $\begin{array}{l}\text { Cortical vein visible } \\
\text { but missed by 4D- } \\
\text { MRA readers }\end{array}$ \\
\hline $\begin{array}{l}\text { Transverse sinus } \\
\text { left }\end{array}$ & IIb & 112 & I & 0 & No & 2017 & 2 & Artery and vein overlay \\
\hline SSS & IIb & 129 & 0 & $\mathrm{Ila}+\mathrm{b}$ & No & 2012 & 3 & $\begin{array}{l}\text { Early drainage due to a } \\
\text { meningioma }\end{array}$ \\
\hline $\begin{array}{l}\text { Cavernous sinus } \\
\text { left }\end{array}$ & III & 0 & III & 0 & No & 2015 & 3 & $\begin{array}{l}\text { Cortical vein visible } \\
\text { but missed by 4D- } \\
\text { MRA readers }\end{array}$ \\
\hline $\begin{array}{l}\text { Posterior fossa } \\
\text { right }\end{array}$ & IV & 43 & III & 0 & No & 2009 & 3 & $\begin{array}{l}\text { Early opacification of } \\
\text { all dural sinuses; } \\
\text { shunt missed due to } \\
\text { misinterpretation of } \\
\text { time sequence in } \\
\text { 4D-MRA }\end{array}$ \\
\hline $\begin{array}{l}\text { Sigmoid sinus } \\
\text { left }\end{array}$ & III & 2 & Ila & 0 & No & 2011 & 2 & $\begin{array}{l}\text { Cortical vein visible } \\
\text { but missed by 4D- } \\
\text { MRA readers }\end{array}$ \\
\hline Medulla & V & 3 & V & 0 & Yes (III/0) & 2011 & 3 & Not visible in the FOV \\
\hline $\begin{array}{l}\text { Parieto-occipital } \\
\text { left }\end{array}$ & III & 85 & III & 0 & No & 2018 & 2 & $\begin{array}{l}\text { Cortical vein visible } \\
\text { but missed by 4D- } \\
\text { MRA readers }\end{array}$ \\
\hline
\end{tabular}

Note:-SSS indicates superior sagittal sinus.

${ }^{a}$ Grading according to Cognard et al. ${ }^{5}$

was considered moderate in terms of the ability to detect a fistula at risk of hemorrhage, with an agreement of $84.3 \%$ and $\kappa=$ 0.6 (95\% CI, $0.3-0.8)$ in a $2 \times 2$ contingency table (low-risk: absence of a shunt and types I-IIa; high-risk: types IIb, III, IV, and $\mathrm{V}$ ) and an agreement of $83.3 \%$ and $\kappa=0.6$ (95\% CI, $0.5-0.7)$ on a 3-tier modified scale (comprising absence of a shunt; types IIIa; types IIb, III, IV, and V).

\section{Diagnostic Value of 4D-MRA Compared with DSA}

The diagnostic accuracy of 4D-MRA in terms of residual/ recurrent DAVF detection yielded a sensitivity of $63.6 \%$ (95\% CI, $40.7 \%-82.8 \%)$, specificity of $96.6 \%$ (95\% CI, $82.2 \%-99.9 \%)$, positive predictive value of $93.3 \%$ (95\% CI,
$68.1 \%-99.8 \%)$, and negative predictive value of $77.8 \%(95 \%$ CI, $60.8 \%-89.9 \%)$. The area under curve score was $0.8(95 \%$ CI, 0.7-0.9).

The diagnostic accuracy of 4D-MRA in terms of bleedingrisk grading yielded a sensitivity of 50\% (95\% CI, 23\%-77\%), specificity of $97.3 \%$ ( $95 \% \mathrm{CI}, 85.8 \%-99.9 \%$ ), positive predictive value of $87.5 \%$ (95\% CI, $47.3 \%-99.7 \%$ ), and negative predictive value of $83.7 \%$ (95\% CI, $69.3 \%-93.2 \%$ ). The area under the curve score was 0.7 (95\% CI, 0.6-0.8).

\section{Retrospective Review of Intermodality Disagreement}

As described in Table 3, intermodality disagreement involved 8 false-negatives and 1 false-positive result. There were several 


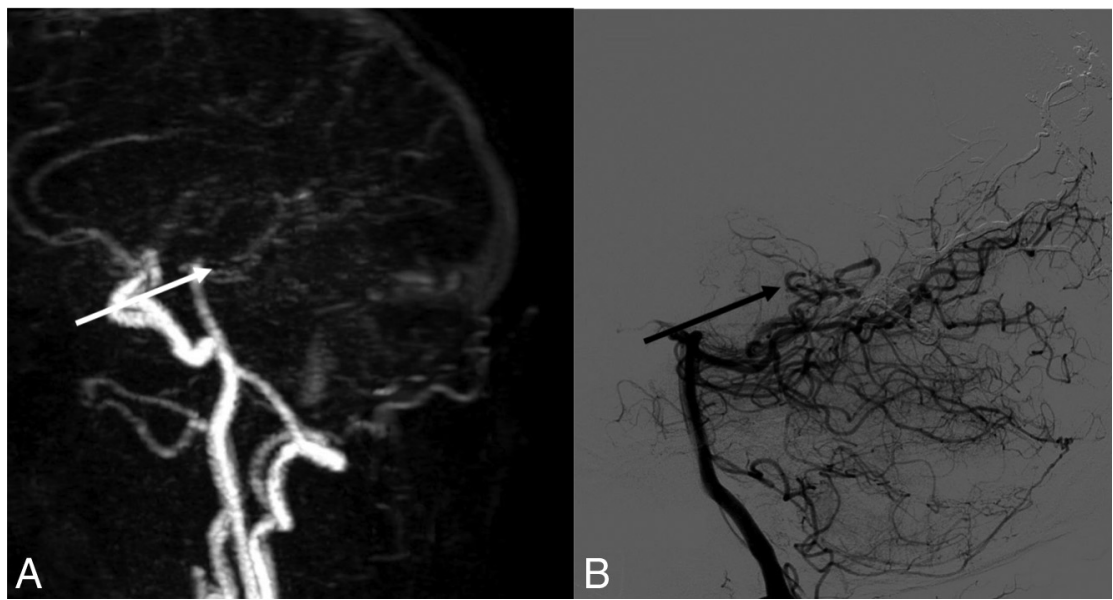

FIG 2. A, Sagittal MIP of 4D-MRA at arterial phase. $B$, Cerebral arteriography through the left vertebral artery in a sagittal view. The white arrow in $A$ and the black arrow in $B$ show early opacification of an epiphyseal vein, before the superior sagittal sinus, confirming an arteriovenous shunt. This examination was rated type III on both imaging modalities.

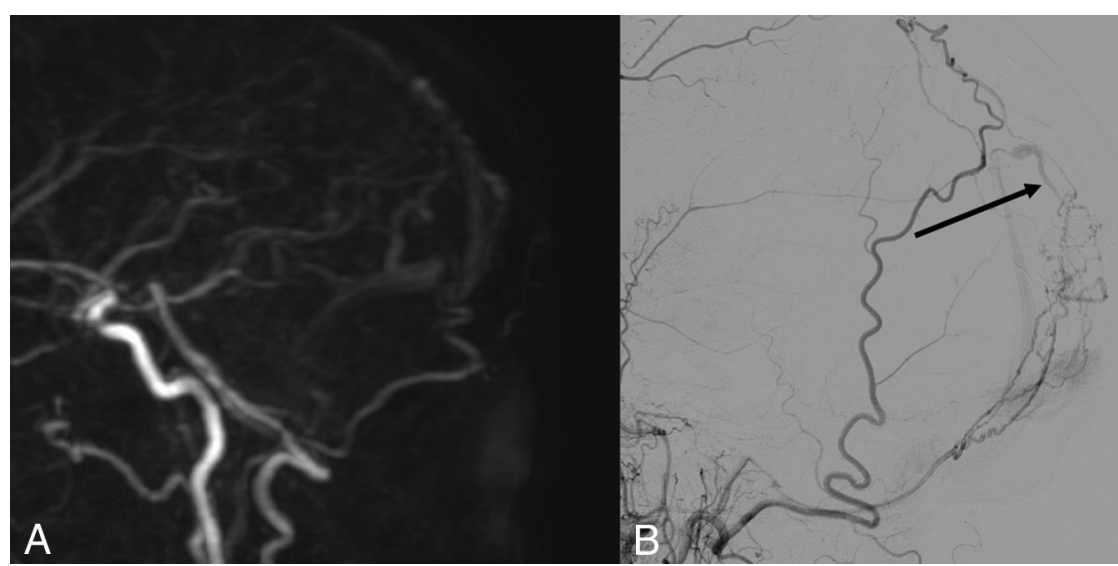

FIG 3. $A$, Sagittal MIP of 4 D-MRA at late arterial phase. $B$, Cerebral arteriography through the left external carotid artery in a sagittal view. The black arrow in $B$ shows early opacification of an occipital vein, confirming an arteriovenous shunt not found in $A$.

DAVF locations: supra- or infra tentorial, near the midsagittal plane, or more laterally. Figs 2 and 3 provide examples of a truepositive result and a false-negative result of $4 \mathrm{D}-\mathrm{MRA}$. Only 1 intermodality disagreement was also an interobserver disagreement. The false-positive case was due to a concomitant condition (early venous drainage due to a meningioma in the vicinity of the DAVF). Six of the 8 false-negative cases involved types III, IV, or V DAVFs, ie, DAVFs with cortical (small) veins. In 4 cases, the cortical vein was shown to be retrospectively present but was missed by all 3 readers.

\section{DISCUSSION}

This study assessed the diagnostic accuracy of 4D-MRA at 3T for detecting any recurrent/residual shunts in treated DAVFs. The sensitivity and specificity of the technique were, respectively, 63.6\% (95\% CI, 40.7\%-82.8\%) and 96.6\% (95\% CI, 82.2\%99.9\%), with substantial intermodality agreement compared with
DSA, which yielded agreement of $82.4 \%$ and $\kappa=0.6$ (95\% CI, $0.4 \%-$ $0.8 \%)$.

$4 \mathrm{D}$ contrast-enhanced MRA is widely used to detect, characterize, and monitor brain vascular malformations and conditions, especially brain arteriovenous malformations. ${ }^{6,8}$ Previous studies reported the diagnostic performance of $4 \mathrm{D}$ MRA for detection and grading of DAVFs. Contrary to brain arteriovenous malformations, only a few studies documented treated DAVFs, and they concerned limited numbers of patients. ${ }^{3,6,9-11}$ The value of 4D-MRA in posttreatment follow-up is, therefore, currently not well-defined. Meckel et $\mathrm{al}^{9}$ evaluated the diagnostic performance of MRA using a timeresolved 3D contrast-enhanced technique with 18 examination pairs ( 9 in a diagnosis group and 9 in a posttreatment follow-up group). On initial diagnosis, both readers identified signs indicative of a DAVF in all 9 cases of angiographically proved fistulas. In the follow-up group (postembolization or surgery), both readers were able to differentiate between complete occlusion of a fistula and a patent residual fistula. However, DAVF occlusion was complete in 5 of the 9 patients. The readers could also use subtracted volumes in addition to 3-plane MIP images, which may have improved diagnostic accuracy. ${ }^{9}$ Bink et $\mathrm{al}^{10}$ reported diagnostic accuracy with 3T MR imaging, with sensitivities and specificities ranging from $84 \%$ to $100 \%$ for 3 readers tasked with detecting DAVFs in 38 patients (19 with DAVFs and 19 without). The readers assessed $4 \mathrm{D}-\mathrm{MRA}$ in addition to 3D-TOF-MRA and 3D-MPRAGE. One patient had undergone endovascular therapy, and 1 patient had undergone surgical closure. ${ }^{10}$ In a consensus reading, Ertl et $\mathrm{al}^{11}$ reported excellent intermodality agreement $(\kappa=1)$ for the pretreatment Cognard classification of lateral DAVFs in 24 patients using additional anatomic images such as TOF-MRA, contrast-enhanced T1-, axial T2-, and axial $\mathrm{T} 2{ }^{\star}$-weighted images. However, there were no type III DAVFs and just 3 type IV DAVFs according to the Cognard classification. Farb et al ${ }^{12}$ compared $4 \mathrm{D}-\mathrm{MRA}$ at $3 \mathrm{~T}$ with DSA for the diagnosis and classification of DAVFs in 42 cases, which included surveillance of a previously cured fistula in 15 cases. In 93\% (39/42) of DAVFs, 3 readers were unanimous and correct in identifying or excluding them. However, all examinations performed for surveillance were negative for DSA, thus limiting the generalizability of the results in this population. 
Unlike in previous studies, we chose to assess 4D-MRA alone without additional morphologic images. ${ }^{10,11}$ This could partly explain the lower performance in this context. Also, posttherapeutic changes, such as embolization product artifacts or anatomic changes, could partly explain these results. Another explanation might be the higher proportion of Cognard type III and IV DAVFs in our study compared with that of Ertl et al. ${ }^{11}$ Indeed, direct drainage into a cortical vein might reduce DAVF detection with 4D-MRA.

The sensitivity and specificity of the technique in terms of the ability to detect a fistula at risk of hemorrhage was, respectively, 50\% (95\% CI, 23\%-77\%) and 97.3\% (95\% CI, 85.8\%-99.9\%), with an agreement of $84.3 \%$ and $\kappa=0.6(95 \% \mathrm{CI}, 0.3-0.8)$ in a $2 \times 2$ contingency table (low-risk: absence of a shunt and types I-IIa; high-risk: types IIb, III, IV, and V) and an agreement of $83.3 \%$ and $\kappa=0.6$ (95\% CI, $0.5-0.7$ ) on a 3-tier modified scale (comprising absence of a shunt; types I-IIa; and IIb, III, IV, and $\mathrm{V})$. Detecting the bleeding risk of a residual/recurrent DAVF is crucial in determining further treatment (false-negative rate of $0.5)$. Six of 8 false-negatives on $4 \mathrm{D}-\mathrm{MRA}$ were types III-V DAVFs. We note that the cortical vein was shown to be retrospectively present but was missed by all 3 readers in 4 cases. For 1 patient, the cortical veins were not included in the FOV. In addition to this acquisition defect, discrepancies could be explained by the lack of spatial or temporal resolution. Also, we might speculate that 3-plane MIP image reading was perhaps not the optimal method. Multiplanar, thin MIP reconstructions could help to alleviate confusion between arteries and veins, though the existing literature on the method for reading 4D-MRA images for DAVF (thick MIP, thin MIP, MPR) is limited.

Interobserver agreement was considered substantial for residual/recurrent DAVF detection, with an agreement of $92 \%$ and $\kappa=0.8\left(95 \%\right.$ CI, 0.6-1). Farb et $\mathrm{al}^{12}$ reported agreement among 3 readers of 0.94 [SD, 0.04] $(P<.001)$. In our study, 4 of 6 cases of disagreement involved type III DAVFs, ie, DAVFs with direct drainage into a cortical vein. All except 1 disagreement involved high-quality (score = 3) 4D-MRA images.

\section{Limitations}

Limitations of our study include its retrospective design and the relatively small number of patients. However, to the best of our knowledge, this is the largest series of patients in the particular case of postembolization evaluation. Indeed, DAVF is a rare disease, and we focused on patients with embolized DAVFs who had undergone DSA and 4D-MRA within a 6-month interval during their follow-up, and this focus reduced the number of eligible patients, possibly resulting in a selection bias because patients with negative findings on DSA or who were asymptomatic but with a residual low-risk DAVF with DSA were less likely to benefit from both modalities within 6 months. The reason for this focus was because it is the first line of treatment for most DAVFs, and surgical treatment may involve the use of materials that can cause artifacts. ${ }^{1}$ Second, different 4D-MRA techniques were used due to the study length and the 2 centers involved. Although Lin et $\mathrm{al}^{13}$ reported a trend toward better performance in newer MR imaging studies, in our cases, intermodality discrepancies involved recent and older examinations, as shown in Table
3. Furthermore, we graded image quality to overcome this limitation. ${ }^{7}$ Third, we read only the 4D-MRA images without any other sequences. This may seem artificial, but it was essential for assessing the diagnostic accuracy of the technique (4D-MRA). Fourth, the statistical distribution of the DAVF types based on the Cognard classification with a higher proportion of true-negative results (DSA, 0; 4D-MRA, 0) was expected but may have influenced the $\kappa$ values.

As previously shown, the use of consensus reading for $4 \mathrm{D}$ MRA in our study improved the diagnostic accuracy and might, therefore, be recommended. ${ }^{12}$ Also, several publications have described novel tools that can further improve 4D-MRA efficacy, opening up new prospects for DAVF assessment before and after treatment. ${ }^{14-17}$ Indeed, venous arterial spin-labeling has shown high sensitivity and specificity in detecting dural arteriovenous fistulas; ${ }^{15,16}$ and the novel temporal spatial acceleration method, HYPRFlow, has also been reported to provide accurate delineation of DAVF vasculature. ${ }^{14}$

\section{CONCLUSIONS}

4D-MRA is a useful noninvasive technique for the follow-up of treated DAVFs. However, given its current limitations, it is not sufficient to confirm an effective cure but can be used as a diagnostic confirmation test. DSA remains mandatory for ensuring optimum bleeding-risk assessment in cases of residual/recurrent DAVF.

Disclosures: François Eugène-UNRELATED: Consultancy: Biomodex. JeanChristophe Gentric-UNRELATED: Consultancy: Medtronic, Stryker, Balt; Stock/ Stock Options: INTRADYS.

\section{REFERENCES}

1. Reynolds MR, Lanzino G, Zipfel GJ. Intracranial dural arteriovenous fistulae. Stroke 2017;48:1424-31 CrossRef Medline

2. Borden JA, Wu JK, Shucart WA. A proposed classification for spinal and cranial dural arteriovenous fistulous malformations and implications for treatment. J Neurosurg 1995;82:166-79 CrossRef Medline

3. Nishimura S, Hirai T, Sasao A, et al. Evaluation of dural arteriovenous fistulas with $4 \mathrm{D}$ contrast-enhanced $\mathrm{MR}$ angiography at $3 \mathrm{~T}$. AJNR Am J Neuroradiol 2010;31:80-85 CrossRef Medline

4. Van Dijk JM, TerBrugge KG, Willinsky RA, et al. Clinical course of cranial dural arteriovenous fistulas with long-term persistent cortical venous reflux. Stroke 2002;33:1233-36 CrossRef Medline

5. Cognard C, Gobin YP, Pierot L, et al. Cerebral dural arteriovenous fistulas: clinical and angiographic correlation with a revised classification of venous drainage. Radiology 1995;194:671-80 CrossRef Medline

6. Soize S, Bouquigny F, Kadziolka K, et al. Value of 4D MR angiography at 3T compared with DSA for the follow-up of treated brain arteriovenous malformation. AJNR Am J Neuroradiol 2014;35: 1903-09 CrossRef Medline

7. Raoult H, Ferré JC, Morandi X, et al. Quality-evaluation scheme for cerebral time-resolved 3D contrast-enhanced MR angiography techniques. AJNR Am J Neuroradiol 2010;31:1480-87 CrossRef Medline

8. Hadizadeh DR, Kukuk GM, Steck DT, et al. Noninvasive evaluation of cerebral arteriovenous malformations by 4D-MRA for preoperative planning and postoperative follow-up in 56 patients: comparison with DSA and intraoperative findings. AJNR Am J Neuroradiol 2012;33:1095-101 CrossRef Medline 
9. Meckel S, Maier M, San Millan Ruiz D, et al. MR angiography of dural arteriovenous fistulas: diagnosis and follow-up after treatment using a time-resolved 3D contrast-enhanced technique. AJNR Am J Neuroradiol 2007;28:877-84 Medline

10. Bink A, Berkefeld J, Wagner $M$, et al. Detection and grading of dAVF: prospects and limitations of 3T MRI. Eur Radiol 2012;22: 429-38 CrossRef Medline

11. Ertl L, Brückmann H, Kunz M, et al. Assessment and treatment planning of lateral intracranial dural arteriovenous fistulas in $3 \mathrm{~T}$ MRI and DSA: a detailed analysis under consideration of timeresolved imaging of contrast kinetics (TRICKS) and ce-MRA sequences. Eur Radiol 2016;26:4284-92 CrossRef Medline

12. Farb RI, Agid R, Willinsky RA, et al. Cranial dural arteriovenous fistula: diagnosis and classification with time-resolved MR angiography at 3T. AJNR Am J Neuroradiol 2009;30:1546-51 CrossRef Medline

13. Lin YH, Lin HH, Liu HM, et al. Diagnostic performance of CT and MRI on the detection of symptomatic intracranial dural arteriovenous fistula: a meta-analysis with indirect comparison. Neuroradiology 2016;58:753-63 CrossRef Medline

14. Clark Z, Johnson KM, Wu Y, et al. Accelerated time-resolved contrast-enhanced magnetic resonance angiography of dural arteriovenous fistulas using highly constrained reconstruction of sparse cerebrovascular data sets. Invest Radiol 2016;51:365-71 CrossRef Medline

15. Amukotuwa SA, Marks MP, Zaharchuk G, et al. Arterial spinlabeling improves detection of intracranial dural arteriovenous fistulas with MRI. AJNR Am J Neuroradiol 2018;39:669-77 CrossRef Medline

16. Iryo $\mathrm{Y}$, Hirai $\mathrm{T}$, Kai $\mathrm{Y}$, et al. Intracranial dural arteriovenous fistulas: evaluation with 3-T four-dimensional MR angiography using arterial spin labeling. Radiology 2014;271:193-99 CrossRef Medline

17. Edjlali M, Roca P, Rabrait C, et al. MR selective flow-tracking cartography: a postprocessing procedure applied to four-dimensional flow MR imaging for complete characterization of cranial dural arteriovenous fistulas. Radiology 2014;270:261-68 CrossRef Medline 